TITLE:

\title{
Propagation of phase-controlled lasers in a two-level medium
}

$\operatorname{AUTHOR}(S)$ :

Nakajima, T

CITATION:

Nakajima, T. Propagation of phase-controlled lasers in a two-level medium. PHYSICAL REVIEW A 2001, 64(4): 043406.

ISSUE DATE:

2001-10

URL:

http://hdl.handle.net/2433/50414

RIGHT:

Copyright 2001 American Physical Society 
PHYSICAL REVIEW A, VOLUME 64, 043406

\title{
Propagation of phase-controlled lasers in a two-level medium
}

\author{
Takashi Nakajima \\ Institute of Advanced Energy, Kyoto University, Gokasho, Uji, Kyoto 611-0011, Japan
}

(Received 6 February 2001; published 13 September 2001)

\begin{abstract}
We study the propagation effects of phase-controlled two-color lasers in a two-level medium under a weak pumping condition. By solving the coupled equations for atoms and fields simultaneously, it is found that the propagation effects significantly modify the atom-field interactions. A time-dependent analysis is also undertaken to explain the numerical results.
\end{abstract}

DOI: 10.1103/PhysRevA.64.043406

PACS number(s): 32.80.Qk, 42.50.Gy, 42.65.-k, 32.80.Rm

\section{INTRODUCTION}

It is now well established, theoretically $[1,2]$ as well as experimentally [3-6], that product yields can be manipulated by controlling the phase difference of two laser fields. When a system is subject to two radiation fields, for example, a fundamental field and its third harmonic, the photoabsorption process can be controlled by externally changing the phase difference between two fields. Needless to say, this process is based on a quantum-mechanical interference caused by three- and single-photon transitions of the fundamental and its third harmonic fields, respectively. Starting from a bound two-level system, the prototype of the phase control scheme, the dynamics have been studied for a few variants such as a bound state and an autoionizing state embedded in a single continuum [7] or multiple continua [8], where the main purpose was to investigate the effect of inherent channel interactions [5,9], represented, for example, by the asymmetric line profile of autoionization and the branching into different continua. The experimental as well as theoretical investigations are not limited to the $\omega-3 \omega$ scheme only. Its basic idea has been applied to the $\omega-2 \omega$ scheme for the control of not only the direction of photoelectron emission [10-12], but also the total ionization yield [13]. We also note that phasesensitive effects by strong short laser fields have also been studied for an atom [14] and molecule [15]. As a different sort of application, we have recently reported the possibility of applying the $\omega-2 \omega$ scheme for a determination of the phase difference of atomic continua [16], in which the phase difference of lasers is used as a "reference" to that of the continua.

It should be noted that most of the related works have been limited, so far, to the response of a single atom or molecule. That is, propagation effects have not been taken into account. Regarding the propagation effects of the phase control scheme, we are aware of only two works. Chen and Elliott [17] reported experimental results from such a viewpoint. More recently Petrosyan and Lambropoulos [18] theoretically examined the propagation effects of phasecontrolled lasers in an optically dense medium of Xe under the strong pumping regime where ac Stark shifts play an important role.

The purpose of this paper is to study the propagation effects of phase-controlled lasers in a two-level medium under a weak pumping regime without ac Stark shifts. Whether the propagation dynamics for the weak pumping regime are similar to those for the strong pumping regime is not a priori obvious, and therefore this work would be complementary to Ref. [18]. It turns out that there are some differences between them as well as some similarities, as described later in this paper. Upon starting this work we had the following questions in mind. First, regarding the properties of the fields, how are the temporal profiles of the phase-controlled pulse pair affected by the medium during the propagation? Can the phase difference be well kept throughout the medium? Obviously these are of essential importance for coherent control. Our second question is associated with the atomic response itself. Since fields drive atoms, and vice versa, the atomic response must necessarily be altered during the propagation if the field properties are altered at all in the medium. Of course the properties of the fields and atoms are related to each other. Therefore, there should be a consistent picture for an understanding of the whole process, and that is what we would like to clarify in this paper.

\section{MODEL}

\section{A. Basic equations}

We consider the system shown in Fig. 1. A laser field with frequency $\omega_{f}$ and its third harmonic with frequency $\omega_{h}\left(=3 \omega_{f}\right)$ interact with a two-level medium. For the complete description of the system, we need equations for atoms and fields. For numerical as well as analytical convenience, we employ a local frame $(\zeta, \tau)$ rather than a lab frame $(z, t)$

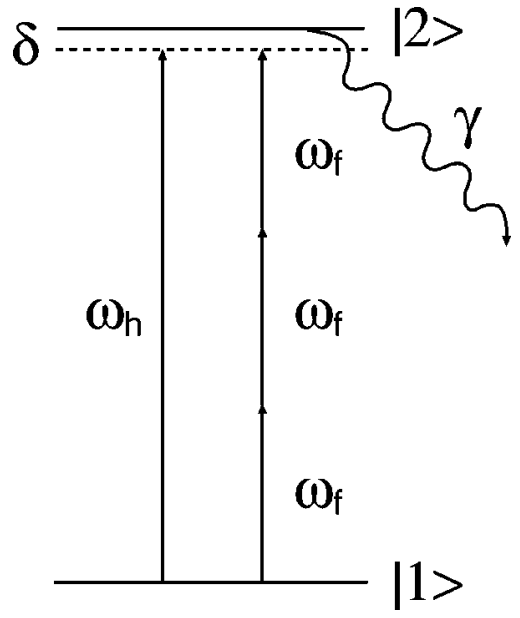

FIG. 1. Level scheme considered in this paper. 
throughout this paper. Needless to say, these two frames are connected through the relations $\tau=t-z / c$ and $\zeta=z$, where $c$ is the speed of light in the vacuum. Now the response of the atom can be described, using amplitude equations, as

$$
\begin{aligned}
& \frac{\partial}{\partial \tau} u_{1}=i\left[\Omega^{(3) *}\left(f(\zeta, \tau)^{*}\right)^{3}+\Omega^{*} h^{*}(\zeta, \tau) e^{i \Delta k \zeta}\right] u_{2}, \\
& \frac{\partial}{\partial \tau} u_{2}=i\left[-\left(\delta-i \frac{\gamma}{2}\right)+\left(S_{2}-S_{1}\right)|f(\zeta, \tau)|^{2}\right] u_{2} \\
& +i\left[\Omega^{(3)}(f(\zeta, \tau))^{3}+\Omega h(\zeta, \tau) \bar{e}^{\Delta k \zeta}\right] u_{1},
\end{aligned}
$$

with $u_{1}$ and $u_{2}$ being the probability amplitudes of states $|1\rangle$ and $|2\rangle$, respectively, and $\delta$ and $\gamma$ the detuning and radiative decay rate out of the system. $\Delta k$ represents wave-vector mismatch between two fields, which is assumed to be zero throughout this paper. $\Omega^{(3)}$ and $\Omega$ are three- and singlephoton Rabi frequencies at peak intensities due to lasers with frequencies $\omega_{f}$ and $\omega_{h}$, respectively. $f$ and $h$ represent complex field amplitudes with frequencies $\omega_{f}$ and $\omega_{h}$, respectively, normalized by the peak field amplitudes at the entrance to the medium. Note that $f$ and $h$ are functions of both time and space. In this paper we limit ourselves to onedimensional propagation along the $\zeta$ axis. $S_{1}$ and $S_{2}$ represent ac Stark shifts of states $|1\rangle$ and $|2\rangle$, at peak intensities of the fundamental field at the entrance to the medium, respectively. The Rabi frequencies $\Omega^{(3)}$ and $\Omega$ and the ac Stark shifts $S_{1}$ and $S_{2}$ can be rewritten, using the peak field amplitudes $\varepsilon_{f 0}$ and $\varepsilon_{h 0}$, and three- and single-photon dipole moments $\mu^{(3)}$ and $\mu$, as

$$
\begin{gathered}
\Omega^{(3)}=\frac{\mu^{(3)} \varepsilon_{f 0}^{3}}{2 \hbar}, \\
\Omega=\frac{\mu \varepsilon_{h 0}}{2 \hbar}, \\
S_{1}=\frac{1}{(2 \hbar)^{2}} \sum_{c_{1}} \frac{\left|\mu_{1 c_{1}} \varepsilon_{f 0}\right|^{2}}{\omega_{c_{1}}-\omega_{1}-\omega_{f}}, \\
S_{2}=\frac{1}{(2 \hbar)^{2}} \sum_{c_{2}} \frac{\left|\mu_{2 c_{2}} \varepsilon_{f 0}\right|^{2}}{\omega_{c_{2}}-\omega_{1}-\omega_{f}},
\end{gathered}
$$

with $\omega_{c_{1}}$ and $\omega_{c_{2}}$ being the energies of nonresonant intermediate states $\left\{\left|c_{1}\right\rangle\right\}$ and $\left\{\left|c_{2}\right\rangle\right\}$. From these equations the polarizations of the medium with frequencies $\omega_{f}$ and $\omega_{h}$ can be obtained, after some algebra, as

$$
\begin{gathered}
P_{f}=2 N\left[2 \hbar\left(s_{1}\left|u_{1}\right|^{2}+s_{2}\left|u_{2}\right|^{2}\right) \varepsilon_{f 0}+3 \mu^{(3)}\left(\varepsilon_{f 0}^{*}\right)^{2} u_{2} u_{1}^{*}\right], \\
P_{h}=2 N \mu u_{2} u_{1}^{*} e^{i \Delta k \zeta},
\end{gathered}
$$

where we have introduced the reduced ac Stark shifts $s_{1}$ $=S_{1} /\left|\varepsilon_{f 0}\right|^{2}$ and $s_{2}=S_{2} /\left|\varepsilon_{f 0}\right|^{2}$ and the atom density $N$.

In order to study the propagation effects, we also need equations for the fields. The wave equations for the fundamental and its third harmonic are found to be written as

$$
\begin{aligned}
& \frac{\partial}{\partial \zeta} f(\zeta, \tau)= i\left(\frac{\omega_{f} N}{c \epsilon_{0} \varepsilon_{f 0}}\right)\left[2 \hbar\left(s_{1}\left|u_{1}\right|^{2}+s_{2}\left|u_{2}\right|^{2}\right) \varepsilon_{f 0} f(\zeta, \tau)\right. \\
&\left.+3 \mu^{(3)}\left(\varepsilon_{f 0}^{*}\right)^{2}\left(f(\zeta, \tau)^{*}\right)^{2} u_{2} u_{1}^{*}\right] \\
& \frac{\partial}{\partial \zeta} h(\zeta, \tau)=i\left(\frac{\omega_{h} N}{c \epsilon_{0} \varepsilon_{h 0}}\right) \mu u_{2} u_{1}^{*} e^{i \Delta k \zeta}
\end{aligned}
$$

where $\epsilon_{0}$ is the permittivity of free space. From these equations it is now clear that the two fields can communicate through the medium. Perhaps it is more precise to say that the harmonic field can communicate with, and is influenced by, the fundamental field, since the right-hand side of Eq. (10) obviously depends on both fundamental and harmonic fields, as can be seen from Eqs. (1) and (2). On the other hand, the fundamental field is hardly affected by the harmonic field, since, although there appears a term containing $u_{2} u_{1}$ on the right-hand side of Eq. (9), it is a higher order term. The dominant contribution on the right-hand side of Eq. (9) comes from the first term which is associated with the induced polarizability.

\section{B. Lowest-order solution of the propagation equations}

For a complete description of the system dynamics including the propagation effects, the equations for the atoms and fields need to be solved self-consistently, which usually requires a numerical elaboration. Under the weak-field limit, however, the whole set of coupled equations given by Eqs. (1), (2), (9), and (10) can be decoupled to some extent, and it turns out that we are left with reduced equations for the fields only, from which analytical solutions can be obtained with further approximations, as we show below. That is, in the weak-field limit, the population of the lower state $|1\rangle$ is practically unity at any time and space, i.e., $u_{1} \sim 1$ for all $(\zeta, \tau)$. Then the amplitude equation for $u_{2}$ becomes

$$
\begin{aligned}
\frac{\partial}{\partial \tau} u_{2}= & i\left[\Omega^{(3)}(f(\zeta, \tau))^{3}+\Omega h(\zeta, \tau)-\left(\delta-i \frac{\gamma}{2}\right)+\left(S_{2}\right.\right. \\
& \left.\left.-S_{1}\right)|f(\zeta, \tau)|^{2}\right] u_{2} .
\end{aligned}
$$

Recalling that the ac Stark shift $S_{2}-S_{1}$ is proportional to the laser intensity of the fundamental field, we may ignore this term under the sufficiently weak pumping where the relation $\gamma \gg\left|S_{2}-S_{1}\right|[19]$, holds. Now we seek for a series expansion solution for $u_{2}$, i.e., let

$$
\begin{aligned}
u_{2}(\zeta, \tau)= & \left(\alpha_{0}(f)^{3}+\alpha_{1} \frac{\partial(f)^{3}}{\partial \tau}+\cdots\right)+\left(\beta_{0} h+\beta_{1} \frac{\partial h}{\partial \tau}\right. \\
& +\cdots),
\end{aligned}
$$

where $\alpha_{0}, \alpha_{1}, \ldots$, and $\beta_{0}, \beta_{1}, \ldots$ are coefficients to be determined. They can be easily determined by substituting Eq. (12) into Eq. (11), resulting in 


$$
\begin{aligned}
& \alpha_{n}=\frac{i^{n} \Omega^{(3)}}{(\delta-i \gamma / 2)^{n+1}} \quad(n=0,1,2, \ldots), \\
& \beta_{n}=\frac{i^{n} \Omega}{(\delta-i \gamma / 2)^{n+1}} \quad(n=0,1,2, \ldots) .
\end{aligned}
$$

Substituting Eq. (12) together with Eqs. (13) and (14) back into Eqs. (9) and (10), we arrive at the reduced equations for the fields,

$$
\begin{gathered}
\frac{\partial}{\partial \zeta} f(\zeta, \tau)=i A_{f} 2 \hbar s_{1} \varepsilon_{f 0} f(\zeta, \tau) \\
\frac{\partial}{\partial \zeta} h(\zeta, \tau)=i A_{h} \mu_{12}\left[\alpha_{0}(f)^{3}+\alpha_{1} \frac{\partial(f)^{3}}{\partial \tau}+\cdots+\beta_{0} h+\beta_{1} \frac{\partial h}{\partial \tau}\right. \\
+\cdots]
\end{gathered}
$$

where

$$
\begin{aligned}
& A_{f}=\frac{\omega_{f} N}{c \epsilon_{0} \varepsilon_{f 0}}, \\
& A_{h}=\frac{\omega_{h} N}{c \epsilon_{0} \varepsilon_{h 0}} .
\end{aligned}
$$

Therefore, in the weak-field limit, the coupled equations (1), (2), (9), and (10) for the atoms and fields can be reduced to the Eqs. (15) and (16) for the fields only. We note that the equation for the fundamental field [Eq. (15)] does not involve the harmonic field. It should also be mentioned that, since we have assumed that the fundamental field is intense and that the three-photon absorption cross section is much smaller than the single-photon one, the fundamental pulse profile is intact during the propagation, as can be seen from Eq. (15). Obviously the solution to Eq. (15) is

$$
f(\zeta, \tau)=f_{0}(\tau) e^{i A_{f} 2 \hbar s_{1} \varepsilon_{f 0} \zeta},
$$

where $f_{0}(\tau)$ is the pulse envelope of the fundamental field at the entrance to the medium, and the exponential term stands for the spatial phase modulation due to the induced polarizability of the lower state $|1\rangle$. Conversely, the propagation of the harmonic field is affected by the fundamental, as can be seen from Eq. (16). However, Eq. (16) is not yet analytically solvable. As the crudest approximation, we ignore all the higher order terms with $\alpha_{n}$ and $\beta_{n}(n \geqslant 1)$ in Eq. (16). After this procedure we finally obtain the lowest-order solution for the harmonic field, which reads

$$
\begin{aligned}
& h(\zeta, \tau)=\left(e^{i \phi_{0}}+\frac{\alpha_{0} A_{h} \mu}{-3 A_{f} 2 \hbar s_{1} \varepsilon_{f 0}+\beta_{0} A_{h} \mu}\right)\left[f_{0}(\tau)\right]^{3} e^{i \beta_{0} A_{h} \mu \zeta} \\
& -\frac{\alpha_{0} A_{h} \mu}{-3 A_{h} 2 \hbar s_{1} \varepsilon_{f 0}+\beta_{0} A_{h} \mu}\left[f_{0}(\tau)\right]^{3} e^{i 3 A_{f} 2 \hbar s_{1} \varepsilon_{f 0} \zeta},
\end{aligned}
$$

where we have introduced $\phi_{0}$ as the initial phase difference of the two fields at the entrance to the medium. In the limit of $\zeta \rightarrow \infty$, the first term $\rightarrow 0$, since $e^{i \beta_{0} A_{h} \mu \zeta} \rightarrow 0$, and hence

$$
\begin{aligned}
h(\zeta & =\infty, \tau) \\
& =-\frac{\alpha_{0} A_{h} \mu}{-3 A_{f} 2 \hbar s_{1} \varepsilon_{f 0}+\beta_{0} A_{h} \mu}\left[f_{0}(\tau)\right]^{3} e^{i 3 A_{f} 2 \hbar s_{1} \varepsilon_{f 0} \zeta} .
\end{aligned}
$$

Therefore, if $\left|-3 A_{f} 2 \hbar s_{1} \varepsilon_{f 0}\right| \ll \beta_{0} A_{h} \mu$,

$$
h(\zeta=\infty, \tau) \sim\left[f_{0}(\tau)\right]^{3}\left(-\frac{\Omega^{(3)}}{\Omega}\right),
$$

which physically means that the asymptotic profile of the harmonic field does not depend on the initial phase difference, and that it becomes identical to that defined at the entrance to the medium, while the phase difference itself approaches a constant value as the pulse pair propagates more deeply into the medium. Obviously if $\Omega$ and $\Omega^{(3)}$ have the same (opposite) sign, a maximum constructive interference occurs at $\phi_{0}=0(\pi)$, and $\phi(\zeta=\infty)=\pi(0)$. Moreover if $\delta=0$ and $\left|3 A_{f} 2 \hbar s_{1} \varepsilon_{f 0} \zeta\right| \ll 1$, Eq. (20) can be further simplified to

$$
h(\zeta, \tau)=\left[\left(e^{i \phi_{0}}+\frac{\Omega^{(3)}}{\Omega}\right) e^{-\left(2 A_{h} \mu \Omega / \gamma\right) \zeta}-\frac{\Omega^{(3)}}{\Omega}\right]\left[f_{0}(\tau)\right]^{3},
$$

which means that, if $\Omega^{(3)}$ and $\Omega$ have the same sign and 0 $<\phi_{0}<\pi$, then $\phi(\zeta=\infty)=\pi-0$. Similarly if $-\pi<\phi_{0}<0$, $\phi(\zeta=\infty)=\pi+0$. This result implies that, although the phase difference tends to become completely out of phase as $\zeta$ $\rightarrow \infty$, which guarantee a maximum destructive interference, the way to approach the constant value depends on whether $\phi_{0}$ is in the upper or lower semisphere of the phase circle.

\section{NUMERICAL RESULTS}

In this section we present representative numerical solutions of the coupled equations given by Eqs. (1), (2), (9), and (10). In order to attain high stability as well as accuracy, the Crank-Nicholson algorithm, which is a hybrid of explicit and implicit methods with second-order accuracy in both space and time, is employed. Before presenting results we describe the various assumptions made for the calculations. The temporal profiles of the two fields at the entrance to the medium are assumed to be Gaussian functions, i.e.,

$$
\begin{gathered}
f(\zeta=0, \tau) \equiv f_{0}(\tau)=\exp \left[-4 \ln 2\left(\frac{\tau}{\tau_{f}}\right)^{2}\right], \\
h(\zeta=0, \tau) \equiv h_{0}(\tau)=\exp \left(i \phi_{0}\right) \exp \left[-4 \ln 2\left(\frac{\tau}{\tau_{h}}\right)^{2}\right],
\end{gathered}
$$

where $\phi_{0}$ is an initial phase difference between two fields at the entrance to the medium, and $\tau_{f}$ and $\tau_{h}$ are the pulse durations (full width at half maximum) of the fundamental 

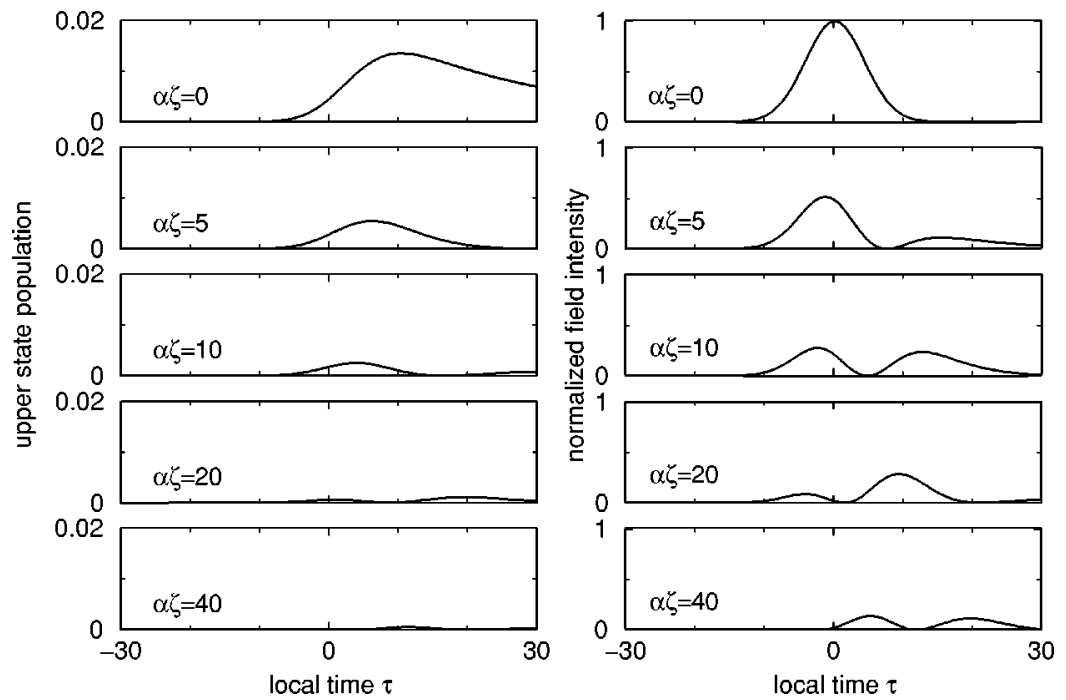

FIG. 2. Variation of the upper state population (left column) and the field amplitudes (right column) as a function of local time $\tau$ at different optical depths $\alpha \zeta=0,5,10,20$, and 40 under the presence of a harmonic field only. The employed parameters are given in the text. and harmonic fields, respectively, with the relation $\tau_{f}$ $=\sqrt{3} \tau_{h}$, which is necessary for the complete temporal overlap of three- and single-photon excitations by the fundamental and harmonic at the entrance to the medium, respectively. It is also assumed that the three- and single-photon Rabi frequencies initially have the same amplitudes, i.e., $\Omega^{(3)}$ $=\Omega$. Obviously these conditions for the pulse profile and Rabi frequencies guarantee that the interference is at a maximum at the entrance to the medium. For the maximum excitation efficiency, the detuning $\delta$ has been taken to be zero. Also, the ac Stark shift term $S_{2}-S_{1}$ has been neglected, assuming $\gamma \gg\left|S_{2}-S_{1}\right|$ under weak pumping, which is usually accompanied by the additional relations $\gamma \gg\left|\Omega^{(3)}\right|,|\Omega|$. This is because $S_{2}-S_{1}$ is second order in $\varepsilon_{f 0}$ while $\Omega^{(3)}$ ( $=\Omega$ in our case) is third order in $\varepsilon_{f 0}$. Now we introduce the absorption coefficients $\alpha$ for the single-photon excitation as

$$
\alpha=4 \Omega A_{h} \mu \gamma^{-1}
$$

As usual, the optical depth for the single-photon excitation can be defined as $\alpha \zeta$. In all the numerical results presented in this paper, we refer to the optical depth with respect to the single-photon process, since, within the optical depth considered here the depletion of the fundamental field does not occur. If we are to describe the system in terms of the parameters $\tau_{f}, \tau_{h}, \Omega^{(3)}, \Omega, \gamma, \delta$, and $\alpha$, the ratio of $A_{h} \mu$ and $3 A_{f} \mu^{(3)}\left(\varepsilon_{f 0}^{*}\right)^{2}$ must be set to a certain value. The reason for this is obvious by comparing Eqs. (9) and (10). Using Eqs. (17) and (18), and the assumption $\mu^{(3)} \varepsilon_{f 0}^{3}=\mu \varepsilon_{h 0}$, which is equivalent to $\Omega^{(3)}=\Omega$, this ratio can be simplified as

$$
\frac{3 A_{f} \mu^{(3)}\left(\varepsilon_{f 0}^{*}\right)^{2}}{A_{h} \mu}=\left(\frac{\varepsilon_{h 0}}{\varepsilon_{f 0}}\right)^{2} \text {. }
$$

A typical ratio of the intensities of both lasers is $10^{-7}-10^{-8}$ for a dipole transition of a neutral atom, and therefore we have specifically chosen the ratio given by Eq. (27) to be $10^{-8}$. We should mention that whether the ratio given in Eq. (27) is $10^{-7}$ or $10^{-8}$ does not make any difference in our numerical results. In summary, the parameters employed for the calculations are $\tau_{f}=10 \sqrt{3}, \tau_{h}=10, \Omega^{(3)}=\Omega=0.01, \gamma$ $=0.04, \delta=0$, and $\alpha=0.1$. We dare not normalize these quantities, for example, with respect to the decay rate $\gamma$, since, by simply considering that they are in units of ns (for $\tau_{h}$ and $\tau_{f}$ ) or $\mathrm{GHz}$ (for $\Omega^{(3)}, \Omega$, and $\gamma$ ), one can obtain some insight into the experimental parameters with ns lasers.

Figure 2 shows the temporal variation, at different optical depths $\alpha \zeta=0,5,10,20$, and 40, of the population in the upper state $|2\rangle$ (left column) and the pulse profile of the harmonic (right column), for the case in which only the harmonic pulse is injected into the medium. Note that the fundamental field is absent. Since the pulse area is rather small, i.e., $\Omega \tau_{h} \sim 0.1$, no Rabi oscillation is seen. The population in the upper state $|2\rangle$ gradually decreases at the deeper optical depth $\alpha \zeta$, which is simply due to the depletion of the harmonic field. A distortion of the pulse shape is also seen. The depletion, however, does not strictly obey Beer's law, since it is a law for a stationary field while we are dealing with a time-varying field. Now we turn on both lasers with an initial phase difference $\phi_{0}=\pi / 3$. The left and right columns of Fig. 3 show, respectively, the temporal variation of the population in the upper state $|2\rangle$, the temporal profiles of the fundamental (right column, dashed line) and harmonic (right column, solid line) pulses, and the phase difference (right column, thin solid line) at different optical depths $\alpha \zeta$ $=0,5,10,20$, and 40 . It is interesting to note that, although the harmonic field is attenuated in the beginning of the propagation $(\alpha \zeta=0 \sim 5)$, it regains its intensity as it propagates further into the medium. Obviously the energy is provided by the fundamental field, which is much more intense than the harmonic field. What is more interesting is that, even after the harmonic field has regained its intensity $(\alpha \zeta>20)$ the upper state is barely populated. In order to understand this, we plot the time evolution of the phase difference at different optical depths, as shown by the thin solid lines in the right column of Fig. 3. It can be seen that, as the pulse pair propagates in the medium, the phase difference tends to become completely out of phase, which implies a perfect destructive interference taking place at large $\alpha \zeta$. This interpretation based on the numerical verification also agrees with our analytical 

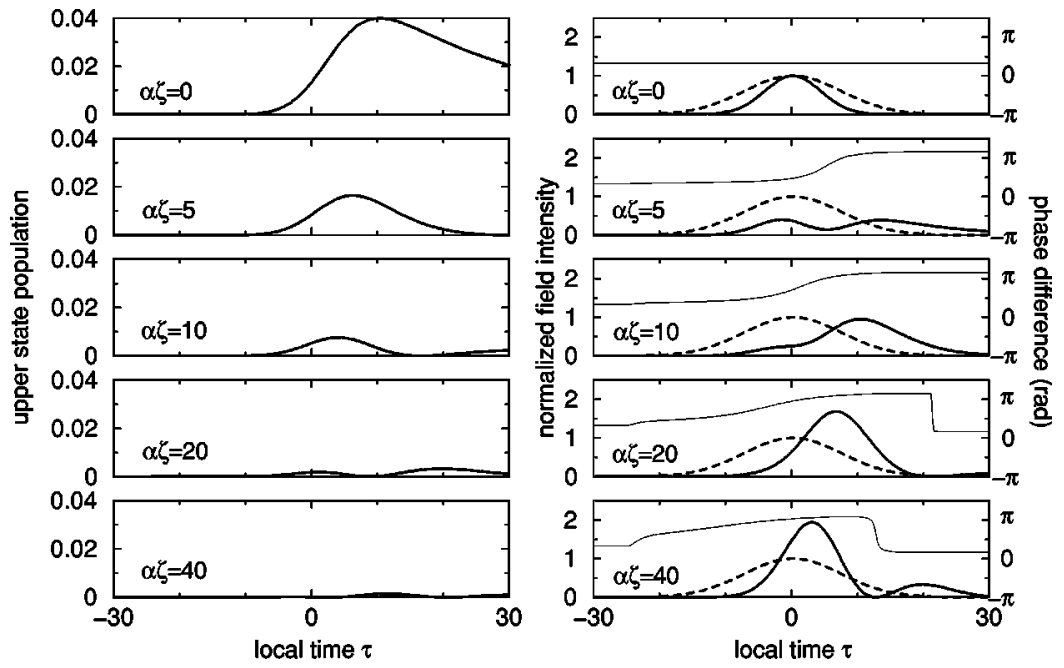

FIG. 3. Variation of the upper state population (left column), and the fundamental (dashed line, right column) and harmonic (solid line, right column) field amplitudes and the phase difference (thin line, right column) as a function of local time $\tau$ at different optical depths $\alpha \zeta=0,5,10,20$, and 40 under the presence of both fundamental and harmonic fields. The phase difference at the entrance to the medium is taken to be $\pi / 3$. The employed parameters are given in the text. result given by Eq. (22). A similar numerical result was also reported in Ref. [18]. Note, however, that the pumping conditions are completely different between them: Here we focus on the weak pumping regime where ac Stark shifts are negligible, while in Ref. [18] they focused on the strong pumping regime where ac Stark shifts are non-negligible. In order to make a direct comparison, we have carried out several computer runs under a strong pumping condition with ac Stark shifts [20], and found that a temporal variation of the phase difference is not smooth but exhibits a rapid oscillation due to the ac Stark shifts, which is quite different from our result. Such a rapid oscillation of the phase difference is implicit in Fig. 2(a) of Ref. [18], but it cannot be directly seen there, since they plotted the phase difference, at the moment when the harmonic field reaches its peak, as a function of propagation distance.

In Fig. 4 we present the temporal pulse profiles of both fields at $\alpha \zeta=160$ for the four different initial phase differences $\phi_{0}=0, \pi / 3,2 \pi / 3$, and $\pi$. At this optical depth, the initial pulse profile of the harmonic field is almost recovered for any $\phi_{0}$. This asymptotic behavior is also what is implied by the lowest order solution given by Eq. (22). Although not shown here, we have assured that the pulse shape becomes

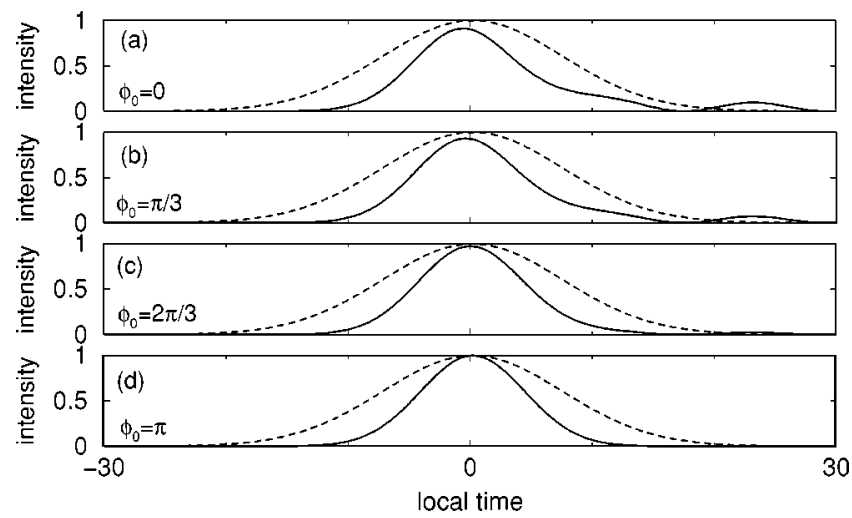

FIG. 4. Temporal profiles of the fundamental (dashed lines) and harmonic (solid lines) fields at the optical depth $\alpha \zeta=160$ as a function of local time. The phase differences employed at the entrance to the medium are (a) 0 , (b) $\pi / 3$, and (c) $2 \pi / 3$, and (d) $\pi$. practically identical for any $\phi_{0}$ at even larger $\alpha \zeta$. Again, this is quite in contrast to the result reported in Fig. 3 of Ref. [18]: Under strong pumping conditions, the ac Stark shifts cannot be ignored, and, as a result, the dynamic detuning varies rapidly in time, leading to the oscillatory behavior of the harmonic pulse profile which never comes back to its original profile, defined at the entrance to the medium, as $\zeta \rightarrow \infty$

We now look at quantities which are perhaps easier to measure, that is, the time-integrated harmonic signal $\int d \tau|h(\zeta, \tau)|^{2}$ and the time- and space-integrated signal defined as $\int d \tau d \zeta\left(1-\left|u_{1}(\zeta, \tau)\right|^{2}\right)$. Figure 5(a) shows the timeintegrated harmonic signal as a function of optical depth for four different initial phase differences $\phi_{0}$. The attenuation of
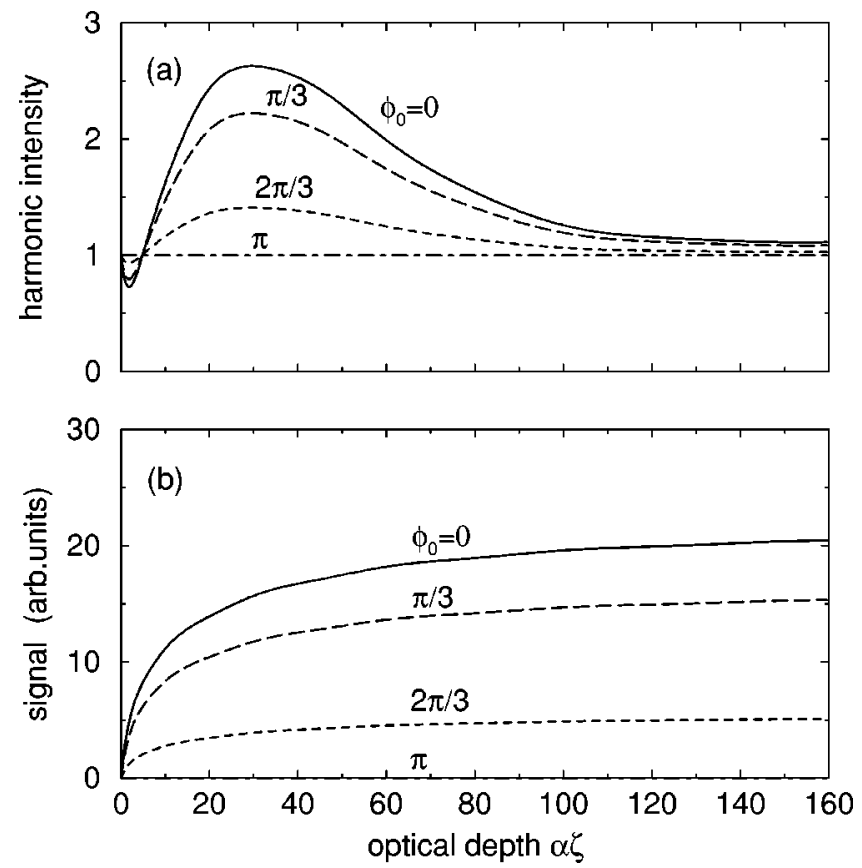

FIG. 5. Variation of the harmonic intensity and the signal as a function of optical depth for different initial phase difference $\phi_{0}$ $=0, \pi / 3,2 \pi / 3$, and $\pi$. (a) Time-integrated harmonic signal. (b) Time- and space-integrated signal. 
the harmonic signal at small depth is followed by an enhancement; then it decreases again, and approaches the original value which is unity. Of course this result is consistent with Figs. 3 and 4. Finally, the time- and space-integrated signal $\int d \tau d \zeta\left(1-\left|u_{1}(\zeta, \tau)\right|^{2}\right)$ is presented in Fig. 5(b) as a function of optical depth for four different initial phase differences $\phi_{0}$. A saturation is observed. Needless to say, this is due to the autoadjustment of the phase difference and the harmonic intensity during the propagation of the pulse pair deeper into the medium, leading to the complete destructive interference at sufficiently large $\alpha \zeta$. This result suggests that, for the purpose of obtaining more signals, the increment of the interaction length along the propagation direction would be of some help up to some point. But a further increase of the interaction length is of no use, since the phase difference and the intensity of the pulse pair are automatically adjusted during the propagation in such a way that a complete destructive interference takes place, contributing to no increase of the signal.

\section{CONCLUSIONS}

In conclusion, we have theoretically studied the propagation effects of phase-controlled two-color lasers in a twolevel medium under a weak pumping condition where ac Stark shifts are negligible. Due to the feedback from atoms to the fields, properties of the third harmonic field are significantly modified during propagation in terms of the temporal pulse shape, amplitude, and phase, while those of the fundamental field are practically unchanged. This is because the fundamental field is much more intense, typically by 7-9 orders of magnitude for a dipole transition of a neutral atom, than the harmonic, resulting in no depletion during propaga- tion. There are two important findings. The first one concerns the phase difference, and the second one the temporal pulse profile of the harmonic field. First, regardless of the initial phase difference between two fields at the entrance to the medium, the pulse pair tends to be completely out of phase after sufficiently long propagation by communicating through the medium. Although the amount of depletion of the harmonic field at the beginning of propagation depends on the initial phase difference, its asymptotic value has no dependence on this. Second, the asymptotic pulse profile of the harmonic field does not depend on the initial phase difference; moreover, it becomes identical to the initial profile defined at the entrance to the medium. These alterations of the harmonic field, in terms of the phase and amplitude, lead to complete destructive interference starting from any initial phase difference. Although the pumping conditions are completely different, some of our findings are qualitatively similar to those in Ref. [18], and suggest that the inclusion of propagation effects is not always favorable for coherent control, since the saturation of the product yield occurs at a certain optical depth. As a final but important remark, we have also carried out a time-dependent analysis in the weakfield limit, and found that the lowest-order solution for the harmonic field explains its asymptotic behavior in terms of the field amplitude and the phase, which of course agrees with the numerical results.

\section{ACKNOWLEDGMENT}

This work was supported by the Grant-in-Aid for scientific research from the Ministry of Education and Science of Japan.
[1] M. Shapiro, J.W. Hepburn, and P. Brumer, Chem. Phys. Lett. 149, 451 (1988).

[2] M. Shapiro and P. Brumer, J. Chem. Soc., Faraday Trans. 93, 1263 (1997).

[3] Ce Chen, Yi-Yian Yin, and D.S. Elliott, Phys. Rev. Lett. 64, 507 (1990); Ce Chen and D.S. Elliott, ibid. 65, 1737 (1990).

[4] Seung Min Park, Shao-Ping Lu, and R.J. Gordon, J. Chem. Phys. 94, 8622 (1991).

[5] L. Zhu, V. Kleiman, X. Li, S. Lu, K. Trentelman, and R.J. Gordon, Science 270, 77 (1995)

[6] X. Wang, R. Bersohn, K. Takahashi, M. Kawasaki, and H.-L. Kim, J. Chem. Phys. 105, 2992 (1996).

[7] T. Nakajima and P. Lambropoulos, Phys. Rev. Lett. 70, 1081 (1993); Phys. Rev. A 50, 595 (1994).

[8] T. Nakajima, J. Zhang, and P. Lambropoulos, J. Phys. B 30, 1077 (1997).

[9] P. Lambropoulos and T. Nakajima, Phys. Rev. Lett. 82, 2266 (1999).
[10] Y.-Y. Yin, C. Chen, D.S. Elliott, and A.V. Smith, Phys. Rev. Lett. 69, 2353 (1992).

[11] E. Dupont, P.B. Corkum, H.C. Liu, M. Buchanan, and Z.R. Wasilewski, Phys. Rev. Lett. 74, 3596 (1995).

[12] H.-L. Kim and R. Bersohn, J. Chem. Phys. 107, 4546 (1997).

[13] E. Papastathopoulos, D. Xenakis, and D. Charalambidis, Phys. Rev. A 59, 4840 (1999).

[14] D.W. Schumacher, F. Weihe, H.G. Muller, and P.H. Bucksbaum, Phys. Rev. Lett. 73, 1344 (1994).

[15] E. Charron, A. Giusti-Suzor, and F.H. Mies, Phys. Rev. Lett. 71, 692 (1993).

[16] T. Nakajima, Phys. Rev. A 61, 041403(R) (2000).

[17] C. Chen and D.S. Elliott, Phys. Rev. A 53, 272 (1996).

[18] D. Petrosyan and P. Lambropoulos, Phys. Rev. Lett. 85, 1843 (2000).

[19] E. Paspalakis, N. J. Kylstra, and P. L. Knight, Phys. Rev. A 60 642 (1999).

[20] T. Nakajima (unpublished). 